\title{
Modélisation moléculaire empirique par Dynamique Moléculaire de molécules de procyanidines B2-diméthyles par le champ de forces SPASIBA
}

\author{
Kicho Denis YAPO $^{1 *}$, Boka Robert N'GUESSAN ${ }^{1}$ et Sébastien Koffi OUFFOUE ${ }^{2}$ \\ ${ }^{I}$ UFR SSMT, Laboratoire de Chimie Organique Structurale, Université Félix Houphouët Boigny, \\ 22 BP 582 Abidjan 22, Côte d'Ivoire. \\ ${ }^{2}$ UFR SSMT, Laboratoire de Chimie Organique Biologique, Université Félix Houphouët Boigny, \\ 22 BP 582 Abidjan 22, Côte d'Ivoire. \\ *Auteur Correspondant ; E-mail: yapokichodenis@yahoo.fr ; Tel: 0022549790419
}

\section{RESUME}

Le champ de forces empiriques SPASIBA nous a permis de modéliser des édifices de type procyanidine B2-diméthyle dans le but d'identifier les structures probables de ces modèles retrouvés dans le milieu intérieur (plasma). Six modèles possibles ont été établis. Ce sont le procyanidine B2-diméthyle 1, le procyanidine B2diméthyle 2, le procyanidine B2-diméthyle 3, le procyanidine B2-diméthyle 4 , le procyanidine B2-diméthyle 5 et le procyanidine B2-diméthyle 6. La simulation par Dynamique Moléculaire s'est faite sur un temps d'une nanoseconde (1ns) avec une température de simulation de 300 Kelvin $(\mathrm{K})$ constante et un volume constant. Les milieux de simulations sont la phase gaz $(\varepsilon=1)$, le milieu biologique implicite $(\varepsilon=4)$ et le milieu hydraté implicite $(\varepsilon=80)$. Les caractéristiques structurales et énergétiques ont montré que les modèles types de molécules procyanidine B2-diméthyl dans le milieu intérieur seraient le procyanidine B2-diméthyle 1 et le procyanidine B2-diméthyle 2 susceptibles d'être synthétisés dans l'optique de réduire éventuellement leur affinité vis-à-vis du fer non héminique $\left(\mathrm{Fe}^{3+}, \mathrm{Fe}^{2+}\right)$ dans l'organisme.

(c) 2014 International Formulae Group. All rights reserved.

Mots clés : Champs de forces SPASIBA, modèles procyanidines B2-diméthyles, Dynamique Moléculaire, simulation, fer non héminique $\left(\mathrm{Fe}^{3+}, \mathrm{Fe}^{2+}\right)$.

\section{INTRODUCTION}

La fonction énergie potentielle empirique ou champ de forces SPASIBA nous a permis de modéliser la complexation du fer non héminique $\left(\mathrm{Fe}^{3+}, \mathrm{Fe}^{2+}\right)$ avec le Procyanidine B2 qui est une molécule de tannin (Yapo et al., 2007; 2008). Cette étude théorique nous a ainsi révélé que la molécule de procyanidine B2 pouvait former, avec le fer non héminique, des complexes avec ses hydroxyles vicinaux. Cette coordination a lieu par l'intermédiaire des groupements hydroxyles vicinaux en position 3' et 4' des proanthocyanidines (Manach. et al., 2004; De Freitas et al., 1998; Tapiero et al., 2002; Brune et al., 1989; Disler et al., 1975). Une des solutions, en vue d'éviter la formation de complexes, est de modéliser les molécules de tannins condensés, le Procyanidine B2 dans le cas spécifique, par occupation des sites de réactions de sorte à empêcher la coordination de celles-ci avec le fer non héminique. Ceci les (fer non héminique, tannins) rendrait disponibles pour l'absorption.

Cette modélisation n'ayant de sens qu'à la condition que les molécules obtenues 
soient assimilables par l'organisme, nous nous proposons de suggérer, par le champ de forces empirique SPASIBA, des modèles dérivées des produits du métabolisme de $O$ diméthylation du Procyanidine B2 retrouvés dans le milieu intérieur (Seigo et al., 2002).

\section{MATERIEL ET METHODES}

\section{Modélisation}

Le Procyanidine B2, qui a servi pour notre étude, est une molécule de tannins condensés de type Proanthocyanidines (Bors et al., 2000). Le squelette structural de ceux-ci (tannins condensés) sert de base aux monomères des Proanthocyanidines comme illustré sur la Figure 1 ci-dessous (Manach et al., 2004; De Freitas et al., 1998; Tapiero et al., 2002; Brune et al., 1989; Disler et al., 1975).

La Figure 2 montre les sites de réaction (sites de coordination pour le fer non héminique) au niveau de la molécule d'étude (Procyanidine B2). Cette molécule est un dimère de monomères de type épicatéchine.<smiles>c1ccc(C2CCc3ccccc3O2)cc1</smiles>

A: cycle phloroglucinol.

B: cycle pyranne.

$\mathrm{C}$ : cycle catéchol pour les procyanidines ou cycle pyrogalloyl dans les prodelphinidines.

Figure 1: Squelette structural de base des monomères de Proanthocyanidines.

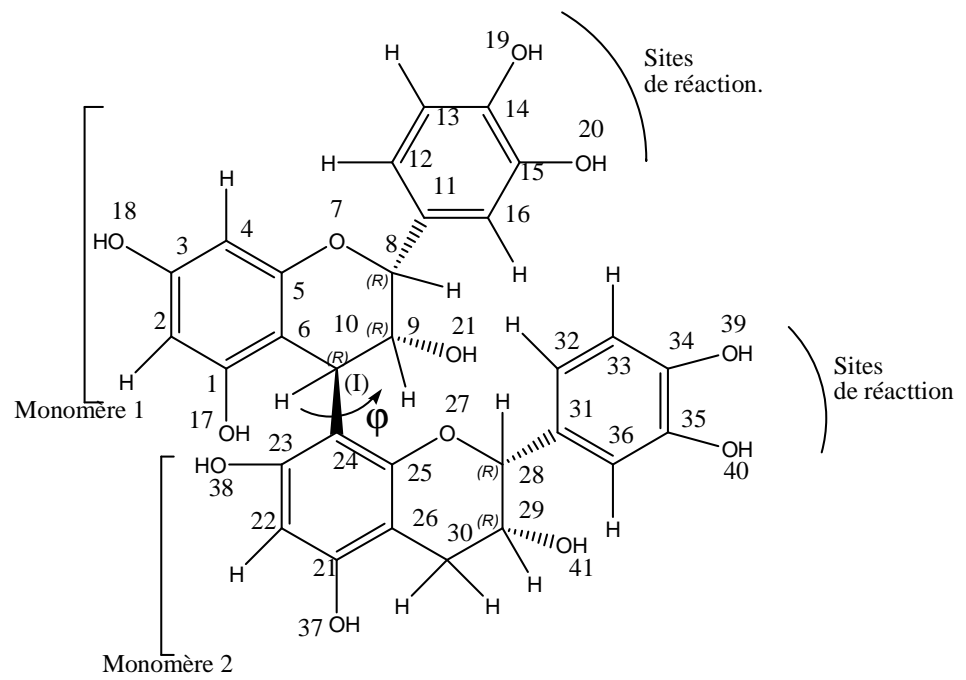

(I): Jonction interflavanique entre le monomère 1 et le monomère 2.

$\varphi$ : Angle dièdre interflavanique entre le monomère 1 et le monomère2.

Figure 2: Molécule de Procyanidine B2 (épicatéchine $(4 \beta \rightarrow 8)$ épicatéchine) ou le $\left(2 \mathrm{R}, 2^{\prime} \mathrm{R}, 3 \mathrm{R}, 3^{\prime} \mathrm{R}\right.$, 4R)-2, 2'-bis (3, 4-dihydroxyphényl)-4, 8'-bichroman-3, 3', 5, 5', 7, 7' hexanol. 
Les réactions de coordination ayant lieu par l'intermédiaire des groupements hydroxyles vicinaux en position 3' et 4' (Figures 1 et 2), le champ de forces SPASIBA nous a permis de prédire des types de complexes susceptibles de se former dans l'organisme (Manach et al., 2004; De Freitas et al., 1998; Tapiero et al., 2002; Brune et al., 1989; Disler et al., 1975; Yapo et al., 2007; 2008). Ce sont des complexes ferreux et ferriques de type Procyanidine B2-Fe ${ }^{2+/ 3+}$ et Procyanidine B2$\mathrm{Fe}^{2+/ 3+}$ - Procyanidine B2. L'occupation des sites de complexation au niveau de la molécule de Procyanidine B2 diminuerait-il l'affinité du fer non héminique ou empêcherait-il tout simplement les phénomènes de complexation? La réponse à cette préoccupation pose un préalable. C'est la modélisation de la dite molécule (Procyanidine B2) en dérivés susceptibles d'être assimilé par l'organisme. L'étude des produits du métabolisme des molécules de tannins condensés ou proanthocyanidines montre que celles-ci subissent dans l'organisme, au niveau intestinal, des $O$ méthylations en positions 3' et/ou 4' (Scalbert et al., 2002; Jeremy, 2003; Seigo et al., 2002;
Roberta et al., 2002). Concernant spécifiquement la molécule de Procyanidine B2, objet de notre étude, Seigo et al. (2002) ont montré que ces molécules contenues en amont dans l'alimentation pouvaient se retrouver en aval dans le milieu intérieur sous forme de molécules de Procyanidine B2 libres, de molécules de procyanidine B2-Ométhylées, de molécules de Procyanidine B2$O$-diméthylées et de monomères, épicatéchines, méthylés et non méthylés (Seigo B. et al., 2002). L'intérêt serait donc de faire une diméthylation sélective de la molécule de procyanidine B2 en positions 3' et 4' au niveau des monomères Seigo et al. (2002) n'ayant pas donné de structures exactes des molécules de Procyanidine B2 $O$ diméthylées retrouvées dans le milieu interieur, la problématique dans cette étude serait donc de modéliser celles-ci par dynamique moléculaire (Seigo et al., 2002).

La $O$-diméthylation en positions 3 ' et 4' des monomères de la molécule de Procyanidine B2 nous donne six possibilités représentées par les structures présentées cidessous (Figures 3 - 8):

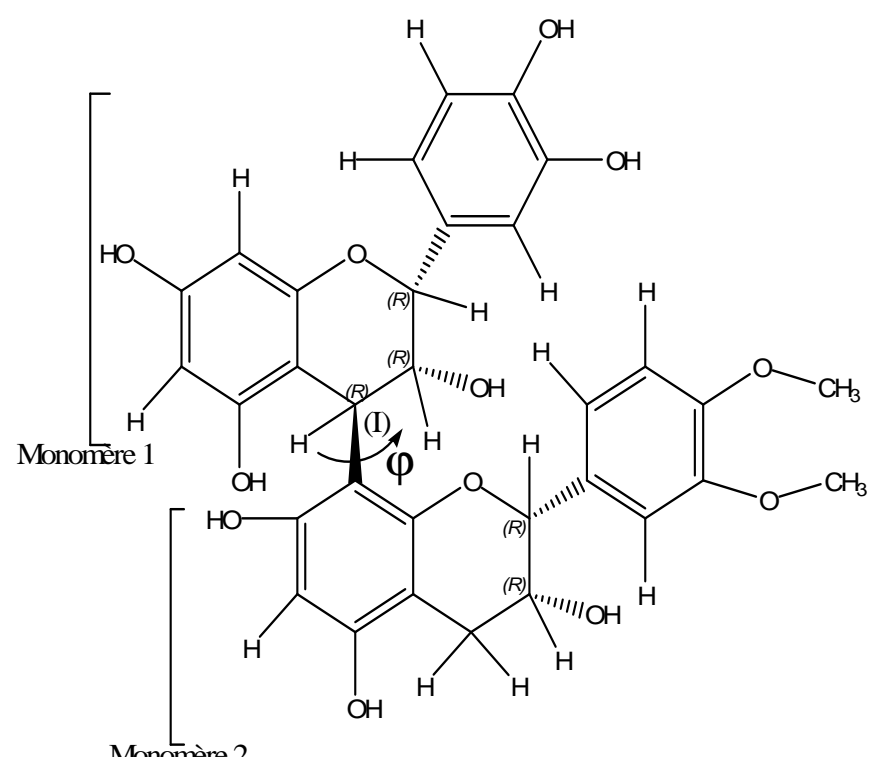

Monomère 2

Figure 3: Procyanidine B2-diméthyle 1.

La molécule de Procyanidine B2-O-diméthylée en positions 3' et 4' du monomère 2. 


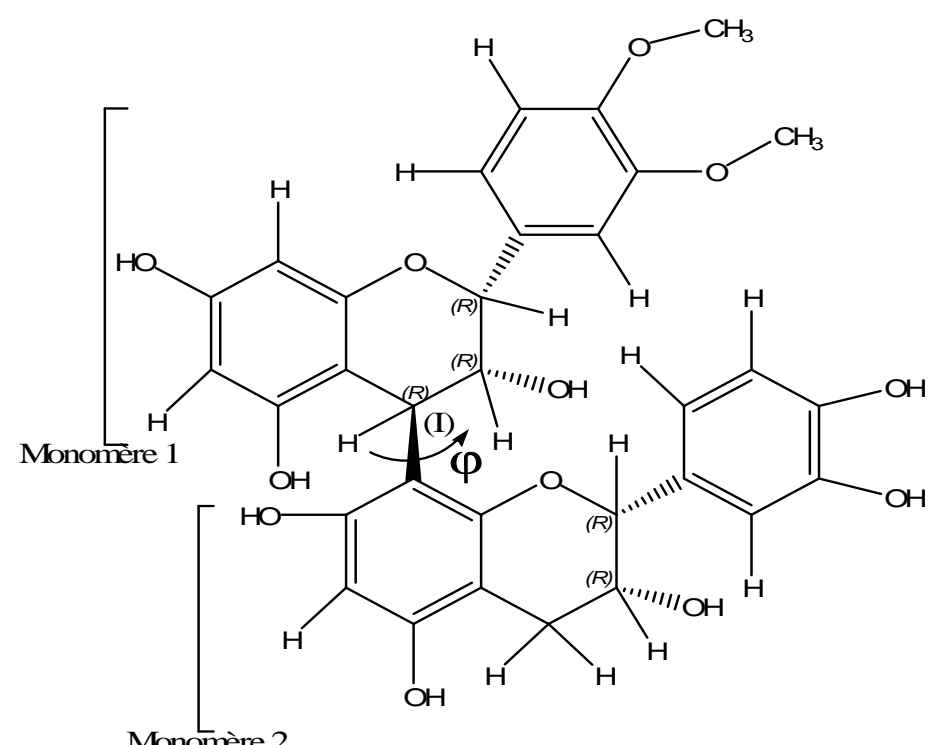

Figure 4: Procyanidine B2-diméthyle 2.

La molécule de Procyanidine B2-O-diméthylée en positions 3' et 4' du monomère 1.

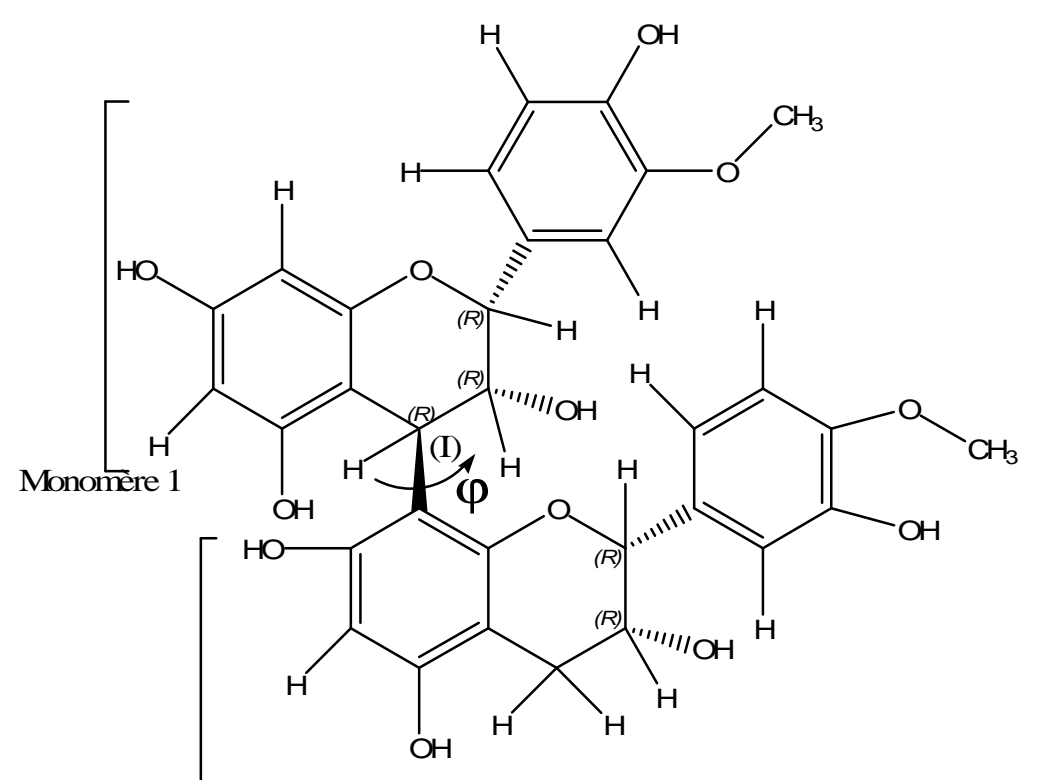

Monomère 2

Figure 5: Procyanidine B2-diméthyle 3.

La molécule de Procyanidine B2-O-diméthylée en positions 3' du monomère 1 et 4' du monomère 2. 


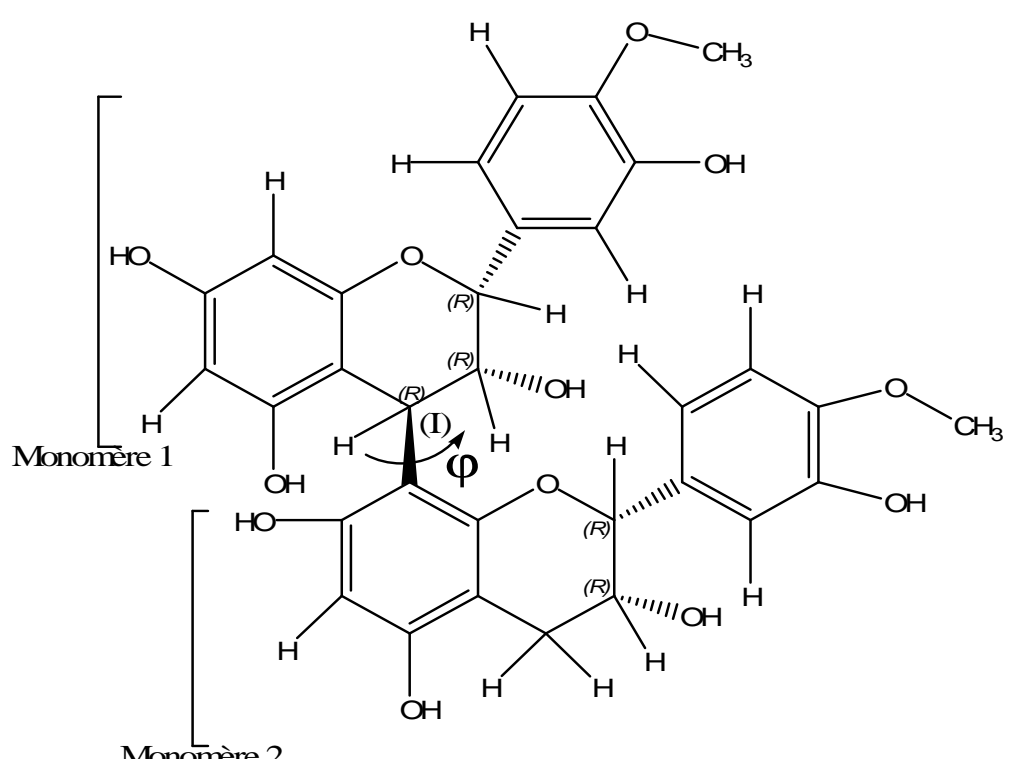

Figure 6: Procyanidine B2-diméthyle 4.

La molécule de Procyanidine B2-O-diméthylée en positions 4' du monomère 1 et 4' du monomère 2.

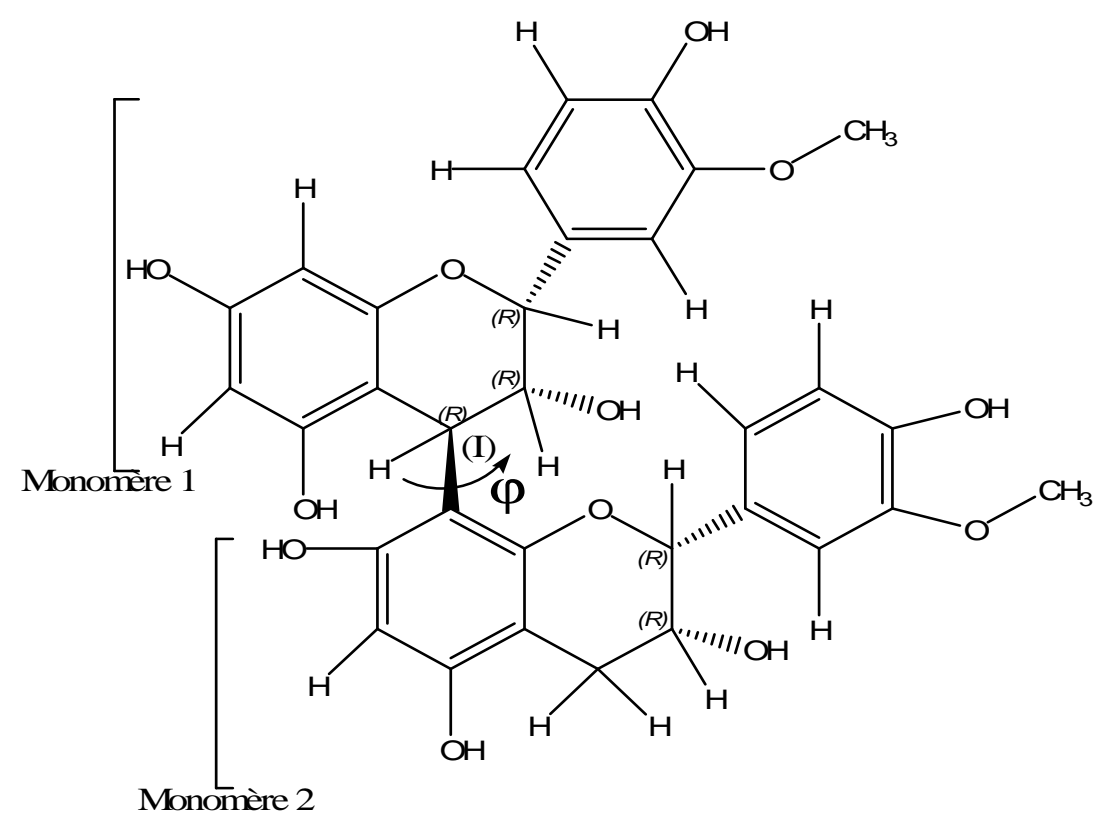

Figure 7: Procyanidine B2-diméthyle 5.

La molécule de Procyanidine B2-O-diméthylée en positions 3' du monomère 1 et 3' du monomère 2. 


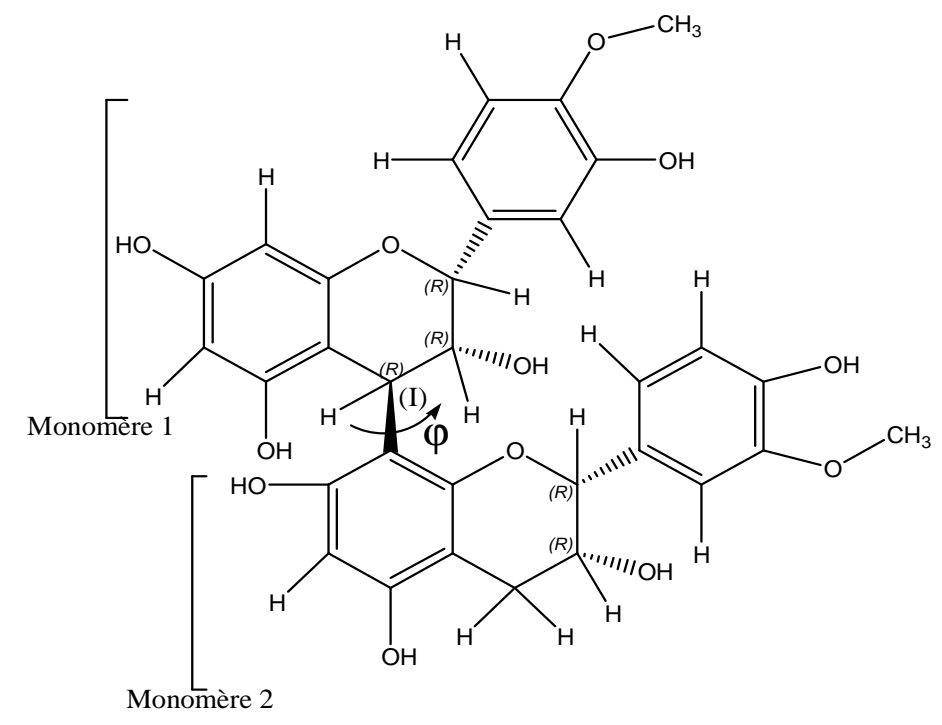

Figure 8: Procyanidine B2-diméthyle 6.

La molécule de Procyanidine B2-O-diméthylée en positions 4' du monomère 1 et 3' du monomère 2.

La modélisation de la structure de Procyanidine B2-O-diméthylée se fera par Dynamique Moléculaire à l'aide du champ de forces empirique SPASIBA.

\section{Champ de forces empiriques SPASIBA}

SPASIBA (Spectroscopic Potential Algorithm for SImulating Biomolecular conformational Adaptability) est une fonction énergie potentielle empirique. Elle combine la fonction énergie potentielle AMBER
$\left(\mathrm{V}_{\mathrm{AMBER}}\right)$ de mécanique moléculaire adaptée aux études conformationnelles et la fonction Urey-Bradley-Shimanouchi $\left(\mathrm{V}_{\mathrm{UBS}}\right)$ adaptée aux études de spectroscopie vibrationnelle Infrarouge et Raman (Weiner et al., 1986; Shimanouchi, 1963; Derman et al., 1977; Siegenberg et al., 1991).

$$
\mathrm{V}_{\text {SPASIBA }}=\mathrm{V}_{\text {AMBER }}+\mathrm{V}_{\mathrm{UBS}}
$$

L'expression de la partie du potentiel provenant d'AMBER $\left(\mathrm{V}_{\mathrm{AM}}\right)$ est la suivante :

$$
\begin{aligned}
& \mathrm{V}_{\mathrm{AM}}=\sum_{\text {dièdres }} \frac{V_{n}}{2}[1+\cos (\mathrm{n} \Phi-\gamma)]+\sum_{n o n l i e ́ s}\left(\frac{A_{i, j}}{r_{i, j}^{12}}-\frac{B_{i, j}}{r_{i, j}^{6}}\right) \\
& (\mathrm{II}) \\
& +\sum_{n \text { nonliés }}\left(\frac{q_{i} q_{j}}{\varepsilon r_{i, j}}\right)+\sum_{\text {liaisonsH }}\left(\frac{C_{i, j}}{r_{i, j}^{12}}-\frac{D_{i, j}}{r_{i, j}^{10}}\right) \\
& \qquad(\mathrm{IIV})
\end{aligned}
$$

\footnotetext{
Le terme I correspond au potentiel de torsion (interactions liantes) ;

Le terme II correspond à des interactions de Van Der Waals ;

Le terme III rend compte de l'énergie potentielle d'origine électrostatique ;

S'il y a une liaison hydrogène entre les atomes i et j, le terme IV est rajouté ;

Les termes II, III et IV représentent les interactions entre atomes non liés ou Potentiel Intermoléculaire.
} 
La partie du potentiel intramoléculaire ou interactions liantes dans le champ de forces SPASIBA est représentée par le potentiel
$\mathrm{V}_{\text {UBS. }}$ Il comprend 4 termes dont les formes sont les suivantes :

$$
\begin{aligned}
& \mathrm{V}_{\mathrm{UBS}}=\mathrm{V}_{1}+\mathrm{V}_{2}+\mathrm{V}_{3}+\mathrm{V}_{4} \\
& \mathrm{~V}_{1}=\sum_{\text {liaisons }} \mathrm{K}\left(\mathrm{r}_{\mathrm{ij}}-\mathrm{r}_{\mathrm{ij} 0}\right)^{2}+\sum_{\text {liaisons }} \mathrm{K}^{\prime} \mathrm{r}_{\mathrm{ij} 0}\left(\mathrm{r}_{\mathrm{ij}}-\mathrm{r}_{\mathrm{ij} 0}\right) \\
& \mathrm{V}_{2}=\sum_{\text {angles }} \mathrm{Hr}_{\mathrm{ij} 0} \mathrm{r}_{\mathrm{jk} 0}\left(\theta_{\mathrm{ij}}-\theta_{\mathrm{ij} 0}\right)^{2}+\sum_{\text {angles }} \mathrm{H}^{\prime} \mathrm{r}_{\mathrm{ij} 0} \mathrm{r}_{\mathrm{jk} 0}\left(\theta_{\mathrm{ij}}-\theta_{\mathrm{ij} 0}\right) \\
& \mathrm{V}_{3}=\frac{1}{2} \sum_{\text {dis tan ces } 1-3} \mathrm{~F}_{\mathrm{ij}}\left(\mathrm{q}_{\mathrm{ij}}-\mathrm{q}_{\mathrm{ij} 0}\right)^{2}+\sum_{\text {dis"tances } 1-3} \mathrm{~F}_{\mathrm{ij}}^{\prime} \mathrm{q}_{\mathrm{ij} 0}\left(\mathrm{q}_{\mathrm{ij}}-\mathrm{q}_{\mathrm{ij} 0}\right)^{2} \\
& \mathrm{~V}_{4}=\mathrm{V}_{1-4 \mathrm{tg}}+\mathrm{V}_{\mathrm{L}}+\mathrm{KapF}_{\mathrm{red}}
\end{aligned}
$$

$\mathrm{V}_{1}$ : potentiel des mouvements d'élongation :

$\mathrm{V}_{2}$ : potentiel de déformation angulaire ;

$\mathrm{V}_{3}$ : potentiel de répulsion 1-3 entre deux atomes liés à un même atome central ;

$\mathrm{V}_{4}$ est caractéristique des groupements méthyle $\mathrm{CH}_{3}$, méthylène $\mathrm{CH}_{2}\left(\mathrm{~V}_{1-4 t g}\right.$ et $\left.\mathrm{V}_{\mathrm{L}}\right)$ et traite la redondance liaisonangle qui apparaît quand un atome central est lié à quatre autres atomes dans l'espace $\left(\mathrm{F}_{\text {red }}\right)$.

L'objectif de l'utilisation de la fonction énergie potentielle SPASIBA est de déterminer des paramètres susceptibles de reproduire les caractéristiques structurales et vibrationnelles d'un système moléculaire en accord avec les données expérimentales et de la mécanique quantique (fonctionnelle de densité en occurrence). Lesquels paramètres vont nous permettre de déterminer par Dynamique Moléculaire le(s) modèle(s) recherché(s) selon la méthodologie ci-après (Yapo et al., 2008).

\section{Méthodologie}

Les caractéristiques énergétiques et conformationnelles obtenues par Dynamique Moléculaire vont nous permettre de juger de la faisabilité de nos modèles. La Dynamique Moléculaire proprement dite est effectuée à volume et température constants. Le temps de simulation est fixé à une nanoseconde (1 ns) en phase gaz et en milieu de solvatation implicite. La température de simulation, après la phase de thermalisation, est de 300 Kelvin (K). Les caractéristiques structurales et énergétiques sont sauvegardées toutes les 0.1 picosecondes $(0.1 \mathrm{ps})$. Les caractéristiques énergétiques permettront de comparer la stabilité relative de ceux-ci. Les caractéristiques conformationnelles détermineront l'éloignement ou non des différentes structures par rapport à la conformation expérimentale de la molécule de procyanidine B2 (Khan et al., 1997). Laquelle conformation expérimentale a pour principale caractéristique la variation de l'angle dièdre interflavanique $\varphi$ entre les deux monomères (Figure 2) se situant autour de $+90^{\circ}$ et $-90^{\circ}$ en solution (Khan et al., 1997). Les six modèles ont chacun une conformation de départ avec un angle dièdre $\varphi$ autour de 98.2 ${ }^{\circ}$. C'est la conformation (structure avec angle dièdre $\varphi=98.2^{\circ}$ ) d'un minimum local de la structure expérimentale de la molécule de Procyanidine B2 (Khan et al., 1997). Tous les modèles seront simulés dans un premier temps, en phase gaz pour suivre leur évolution respective sans aucune contrainte. Puis, ils seront étudiés en solvatation implicite en milieu biologique $(\varepsilon=4)$ et en milieu hydraté ( $\varepsilon=80)$ pour constater les comportements respectifs des modèles par application d'un champ diélectrique continu (Still et al., 1990). Enfin les modèles en phase gaz de la Dynamique Moléculaire seront comparés aux 
modèles résultant de calcul théorique B3LYP/6-311G également en phase gaz.

\section{RESULTATS}

Notons qu'un modèle de Procyanidine B2 $O$-dimethylé viable est un modèle avec une conformation dont l'angle dièdre $\varphi$ entre les deux monomères constitutifs se rapproche le plus possible de + $90^{\circ} \mathrm{ou}-90^{\circ}$ et une caractéristique énergétique la plus basse possible par rapport aux autres modèles.

\section{Caractéristiques énergétiques}

Les caractéristiques énergétiques des modèles de procyanidine B2 $O$-diméthylée, matérialisées par les énergies potentielles, sont données dans le Tableau 1.

- En phase gaz, considérant l'aspect énergétique, le modèle Procyanidine.B2diméthyle 2 avec une $\mathrm{E}_{\mathrm{p}}$ de $-36.42 \mathrm{kcal}^{\mathrm{mol}}{ }^{-1}$ serait relativement plus stable que les autres. Le modèle Procyanidine B2-diméthyle 3 qui a une énergie potentielle de - $7.49 \mathrm{kcal}_{\mathrm{mol}}{ }^{-1}$, serait la moins stable.

- En milieu biologique implicite, les modèles de procyanidine B2 $O$-diméthylée sont tous dans des états de stabilité plus ou moins proches d'autant plus que les énergies potentielles sont comprises entre 62 et 72 $\mathrm{kcal}^{\mathrm{mol}}{ }^{-1}$. Néanmoins, on peut dire que le modèle Procyanidine B2-diméthyle 5, avec une $E_{p}$ de $62.90 \mathrm{Kcal}_{\mathrm{mol}}{ }^{-1}$, est relativement dans un état de meilleure stabilité suivi du modèle Procyanidine B2-diméthyle 2 avec une $\mathrm{E}_{\mathrm{p}}$ de $67.94 \mathrm{kcal}^{\mathrm{mol}}{ }^{-1}$.

- En milieu hydraté implicite, les énergies potentielles respectives des modèles indiquent des états énergétiques semblables aux environs de $95 \mathrm{kcal}^{\mathrm{mol}}{ }^{-1}$, signifiant un même niveau de stabilité relative.

Les RMSD (Ecart Type), se situant autour de 5, indiquent de faibles variations autour des moyennes des énergies potentielles des modèles pendant les dynamiques.

\section{Caractéristiques conformationnelles}

Les variations conformationnelles des modèles de procyanidine B2 $O$ diméthylée, par fluctuation de l'angle dièdre $\varphi$ de la molécule de Procyanidine B2 au niveau de chaque modèle, sont consignées dans le Tableau 2.

Tableau 1: Energie potentielle $\left(\mathrm{E}_{\mathrm{P}}\right)$ en $\mathrm{kcal}^{\mathrm{mol}}{ }^{-1}$ des modèles de procyanidine B2 $O$-diméthylée dans les milieux de simulation.

\section{EP $_{P} \pm$ RMSD (Ecart Type) en Milieux de simulation}

\begin{tabular}{lccc}
\multicolumn{1}{c}{ Modèles } & Phase gaz & $\begin{array}{c}\text { Milieu biologique } \\
\text { implicite }\end{array}$ & $\begin{array}{c}\text { Milieu hydraté } \\
\text { implicite }\end{array}$ \\
\hline Procyanidine B2-diméthyle 1 & $-24.54 \pm 5.11$ & $69.78 \pm 4.90$ & $95.98 \pm 5.02$ \\
\hline Procyanidine B2-diméthyle 2 & $-36.42 \pm 6.07$ & $67.94 \pm 4.82$ & $95.37 \pm 5.06$ \\
\hline Procyanidine B2-diméthyle 3 & $-7.49 \pm 4.99$ & $72.24 \pm 5.02$ & $97.13 \pm 4.94$ \\
\hline Procyanidine B2-diméthyle 4 & $-18.89 \pm 5.14$ & $71.08 \pm 4.95$ & $96.20 \pm 5.02$ \\
\hline Procyanidine B2-diméthyle 5 & $-15.63 \pm 5.12$ & $62.90 \pm 3.86$ & $93.59 \pm 5.17$ \\
\hline Procyanidine B2-diméthyle 6 & $-23.65 \pm 4.89$ & $71.23 \pm 5.06$ & $95.23 \pm 5.10$ \\
\hline
\end{tabular}


Tableau 2: Variation moyenne du dièdre $\varphi$ en degré $\left({ }^{\circ}\right)$ des modèles de procyanidine B2 $O$ diméthylée dans les milieux de simulations.

\begin{tabular}{lccc}
\hline \multirow{2}{*}{ Modèles } & \multicolumn{3}{c}{ Dièdre $\varphi$ moyen en degré $\left(^{\circ}\right) \pm$ RMSD (Ecart Type) } \\
& \multicolumn{3}{c}{ En milieux de simulation } \\
\cline { 2 - 4 } & Phase gaz & $\begin{array}{c}\text { Milieu biologique } \\
\text { implicite }\end{array}$ & $\begin{array}{c}\text { Milieu hydraté } \\
\text { implicite }\end{array}$ \\
\hline $\begin{array}{l}\text { Procyanidine B2- } \\
\text { Diméthyle 1 }\end{array}$ & $92.33 \pm 13.88$ & $90.91 \pm 11.88$ & $91.26 \pm 11.97$ \\
\hline $\begin{array}{l}\text { Procyanidine B2- } \\
\text { Diméthyle 2 }\end{array}$ & $91.58 \pm 16.77$ & $91.03 \pm 11.87$ & $93.51 \pm 12.30$ \\
\hline $\begin{array}{l}\text { Procyanidine B2- } \\
\text { Diméthyle 3 }\end{array}$ & $88.62 \pm 14.36$ & $94.01 \pm 13.31$ & $-108.86 \pm 14.73$ \\
\hline $\begin{array}{l}\text { Procyanidine B2- } \\
\text { Diméthyle 4 }\end{array}$ & $87.05 \pm 13.54$ & $9077 \pm 12.33$ & $92.10 \pm 11.97$ \\
\hline $\begin{array}{l}\text { Procyanidine B2- } \\
\text { Diméthyle 5 }\end{array}$ & $-83.87 \pm 16.61$ & $91.81 \pm 9.28$ & $-83.98 \pm 17.34$ \\
\hline $\begin{array}{l}\text { Procyanidine B2- } \\
\text { Diméthyle 6 }\end{array}$ & $89.96 \pm 14.42$ & $-84.53 \pm 15.77$ & $88.90 \pm 14.08$ \\
\hline
\end{tabular}

- En phase gaz, les moyennes s'établissent autour de $90^{\circ}$ pour tous les modèles à l'exception du Procyanidine B2diméthyle $5\left(-84^{\circ}\right)$.

- En milieu biologique implicite, les modèles ont des angles $\varphi$ qui varient autour de $90^{\circ}$ sauf celui du Procyanidine B2-diméthyle 6 qui a une moyenne de $-85^{\circ}$.

- En milieu hydraté implicite, la variation dièdrale est de $90^{\circ}$ en moyenne pour les modèles Procyanidine B2-diméthyle 1, Procyanidine B2-diméthyle 2, Procyanidine B2-diméthyle 4 et Procyanidine B2-diméthyle 6. Les modèles Procyanidine B2-diméthyle 3 et Procyanidine B2-diméthyle 5 ont les leurs respectivement autour de $-109^{\circ}$ et $-84^{\circ}$.

Les RSMD (Ecart Type) montrent qu'il y a des fluctuations plus ou moins importantes autour des moyennes de variations dièdrales des modèles dans les différents milieux de simulations.

Les Figures 9, 10 et 11 montrent les fluctuations simultanés des énergies et des angles dièdres des différents modèles dans les milieux de simulations.
Les modèles viables, c'est-à-dire susceptibles d'être retrouvées dans le milieu intérieur, sont ceux qui présentent à la fois un bas niveau énergétique et une conformation en accord avec l'expérimentation (Khan et al., 1997). Comme le montrent les Figures 9, 10 et 11, le Procyanidine B2-Diméthyle 2 a, en phase gaz (absence de contraintes sur les édifices), l'énergie la plus basse et une conformation dont l'angle dièdre est autour de 90 degré. Dans cette logique, on a juste après le Procyanidine B2-Diméthyle 1. Dans les milieux biologique et hydraté simulés, nous avons pour ces deux modèles (Procyanidine B2-Diméthyle 2 et Procyanidine B2Diméthyle 1), des niveaux énergétiques parmi les plus bas et des angles dièdres proches de l'expérimentation. Pour conforter ces résultats de Dynamique Moléculaire, nous avons effectué des calculs de chimie quantique en phase gaz (milieu sans contrainte) avec la méthode de fonctionnelle de densité (DFT) B3LYP/6-311G appliqués aux modèles étudiés. 


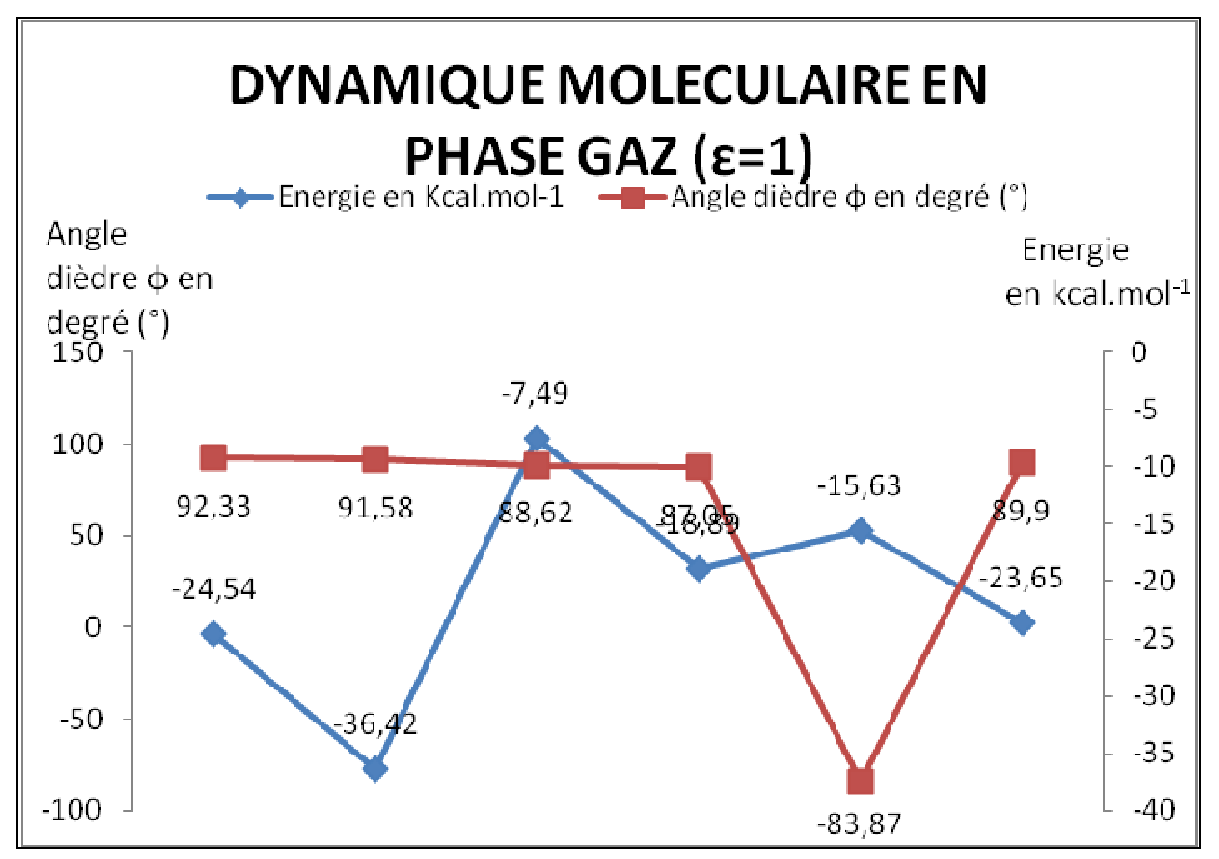

Figure 9: Variation des énergies et des angles dièdres pendant la dynamique moléculaire d'une nanoseconde (1 ns) en phase gaz.

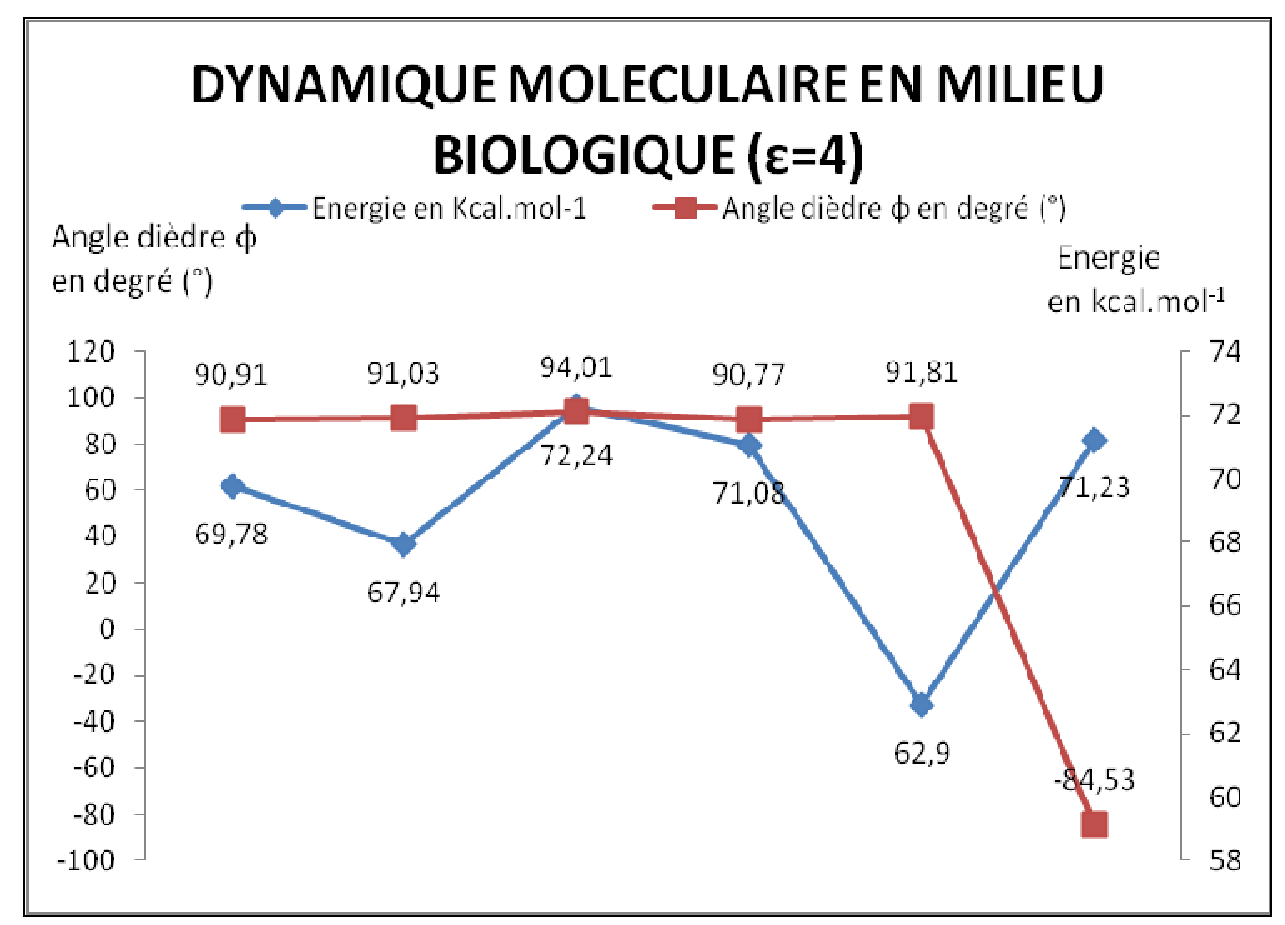

Figure 10: Variation des énergies et des angles dièdres pendant la dynamique moléculaire d'une nanoseconde ( $1 \mathrm{~ns}$ ) en milieu biologique. 
Le Tableau 3 nous donne les énergies associées aux angles dièdres des différents modèles en densité B3LYP/6-311G. La Figure 12 montre la variation de l'angle dièdre en fonction de l'énergie en B3LYP/6-311G. Le Tableau 4 nous donne les longueurs de liaison, les angles de liaison et les angles dièdres des modèles en Dynamique Moléculaire et en mécanique quantique B3LYP/6-311G comparés aux données expérimentales (Fox et al., 1995). Ce qui nous permettra de vérifier l'effet Jahn Teller (Toute molécule non linéaire dans un état électronique dégénéré (orbitale ou spin) se déforme de manière à diminuer sa symétrie, lever la dégénérescence et minimiser son énergie). La Figure 13 nous montre quant à elle, la corrélation des angles dièdres des modèles calculés par B3LYP/6-311G et par Dynamique Moléculaire.

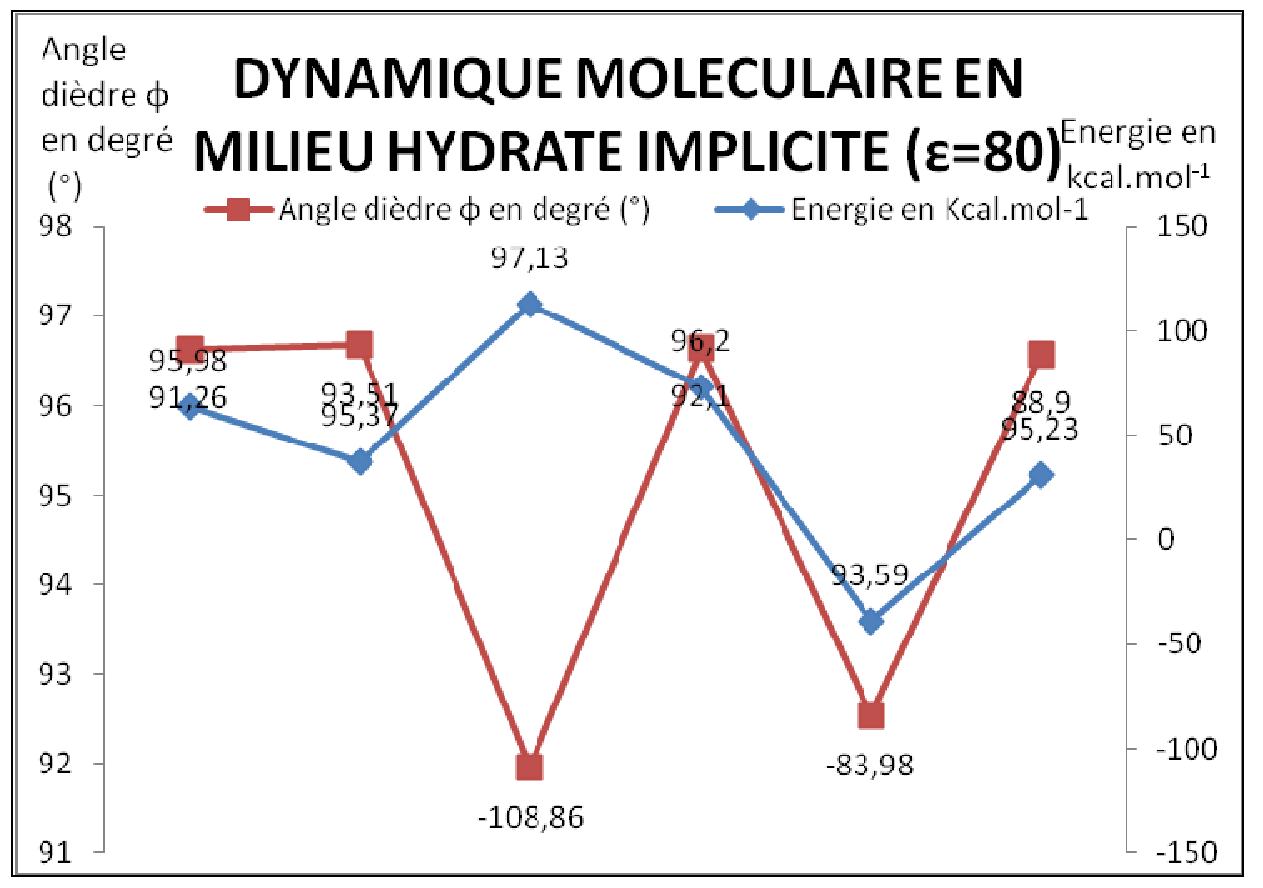

Figure 11: Variation des énergies et des angles dièdres pendant la dynamique moléculaire d'une nanoseconde (1 ns) en milieu hydraté implicite.

Tableau 3: Energie en $\mathrm{kcal}_{\mathrm{mol}}{ }^{-1}$ et dièdre $\varphi$ en degré $\left(^{\circ}\right)$ des modèles de procyanidine B2 $O$-diméthylée en phase gaz.

\begin{tabular}{ccc}
\hline Modèles & Energie en kcal.mol ${ }^{-1}$ & Angle dièdre $\varphi$ en degré $\left(^{\circ}\right)$ \\
\hline Procyanidine B2-diméthyle 1 & $-1342899.34 \pm 0.0057$ & 92.50 \\
Procyanidine B2-diméthyle 2 & $-1342893.84 \pm 0.0142$ & 88.31 \\
Procyanidine B2-diméthyle 3 & $-1342896.15 \pm 0.0043$ & 93.37 \\
Procyanidine B2-diméthyle 4 & $-1342892.14 \pm 0.0007$ & 92.71 \\
Procyanidine B2-diméthyle 5 & $-1342895.29 \pm 0.0390$ & 95.80 \\
Procyanidine B2-diméthyle 6 & $-1342895.87 \pm 0.0042$ & 61.82 \\
\hline
\end{tabular}




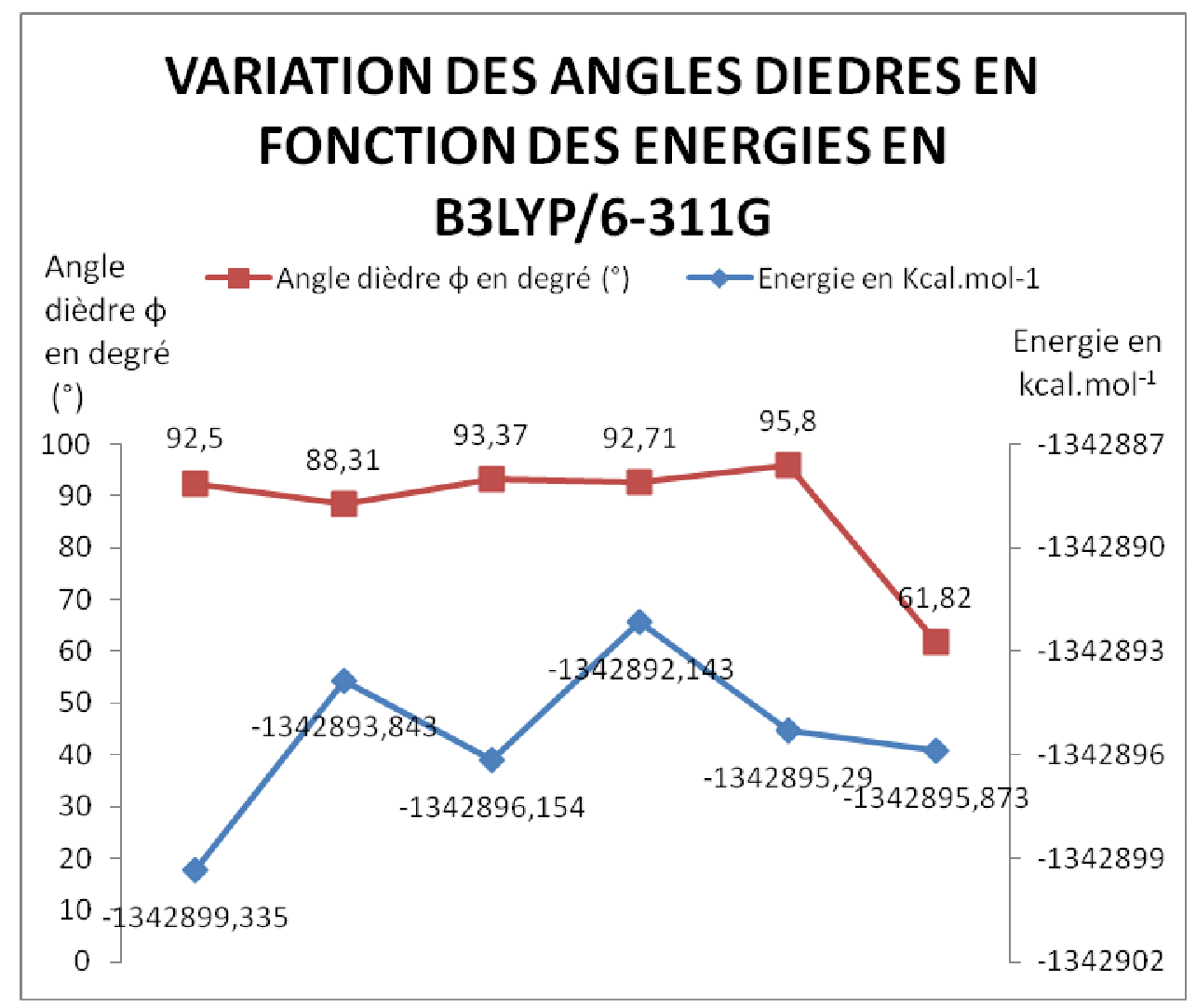

Figure 12: Variation des énergies et des angles dièdres en B3LYP/6-311G.

En phase gaz, nous remarquons que la plus grande différence entre les énergies des différents modèles est de $6 \mathrm{kcal}^{\mathrm{mol}} \mathrm{l}^{-1}$ (Tableau 3, Figure 12). Ce qui nous signale une stabilité énergétique plus ou moins identique. Néanmoins, nous pourrons affirmer que le modèle Procyanidine B2-diméthyle 1 avec une énergie de $-1342899.34 \mathrm{kcal}^{\mathrm{mol}}{ }^{-1}$ est la plus stable de tous. Quand aux angles dièdres, nous constatons que les modèles Procyanidine B2-diméthyle 1 et Procyanidine B2-diméthyle 2 sont ceux dont les mesures dièdres sont les plus proches de $90^{\circ}$ par rapport aux autres modèles. Ce qui confirme les résultats de nos simulations en Dynamique Moléculaire avec le champ de forces SPASIBA comme le montre la Figure 12. 


\section{K. D. YAPO et al. / Int. J. Biol. Chem. Sci. 8(1): 355-376, 2014}

Tableau 4: Longueurs de liaison, angles de liaison et angles dièdres des modèles en Dynamique Moléculaires et en mécanique quantique B3LYP/6-311G.

\begin{tabular}{|c|c|c|c|c|c|c|c|}
\hline \multirow{2}{*}{ Liaisons $(\AA)$} & \multicolumn{2}{|c|}{ Procyanidine B2-Diméthyle 1} & \multicolumn{2}{|c|}{ Procyanidine B2-Diméthyle 2} & \multicolumn{2}{|c|}{ Procyanidine B2-Diméthyle 3} & \multirow{2}{*}{ Exp. } \\
\hline & $\begin{array}{l}\text { Dynamique } \\
\text { Moléculaire }\end{array}$ & $\begin{array}{c}\text { B3LYP/ } \\
6-311 G\end{array}$ & $\begin{array}{l}\text { Dynamique } \\
\text { Moléculaire }\end{array}$ & $\begin{array}{c}\text { B3LYP/ } \\
6-311 G\end{array}$ & $\begin{array}{l}\text { Dynamique } \\
\text { Moléculaire }\end{array}$ & $\begin{array}{c}\text { B3LYP/ } \\
6-311 G\end{array}$ & \\
\hline $\mathrm{C} 1-\mathrm{C} 2$ & 1.41 & 1.39 & 1.44 & 1.39 & 1.39 & 1.39 & 1.40 \\
\hline $\mathrm{C} 2-\mathrm{C} 3$ & 1.38 & 1.40 & 1.40 & 1.39 & 1.40 & 1.39 & - \\
\hline $\mathrm{C} 3-\mathrm{C} 4$ & 1.39 & 1.39 & 1.35 & 1.39 & 1.39 & 1.39 & - \\
\hline $\mathrm{C} 4-\mathrm{C} 5$ & 1.40 & 1.39 & 1.37 & 1.39 & 1.40 & 140 & - \\
\hline $\mathrm{C} 5-\mathrm{C} 6$ & 1.38 & 1.40 & 1.41 & 1.40 & 1.34 & 1.40 & - \\
\hline C1-C6 & 1.40 & 1.40 & 1.41 & 1.41 & 1.41 & 1.41 & - \\
\hline $\mathrm{C} 11-\mathrm{C} 12$ & 1.34 & 1.40 & 1.43 & 1.40 & 1.40 & 1.40 & - \\
\hline C12-C13 & 1.39 & 1.40 & 1.36 & 1.40 & 1.35 & 1.40 & - \\
\hline C13-C14 & 1.43 & 1.39 & 1.39 & 1.40 & 1.40 & 1.39 & - \\
\hline C14-C15 & 1.37 & 1.40 & 1.37 & 1.41 & 1.40 & 1.41 & - \\
\hline C15-C16 & 1.39 & 1.39 & 1.42 & 1.391 & 1.39 & 1.39 & - \\
\hline C11-C16 & 1.44 & 1.41 & 1.40 & 1.41 & 1.41 & 1.41 & - \\
\hline $\mathrm{C} 21-\mathrm{C} 22$ & 1.35 & 1.39 & 1.43 & 1.39 & 1.39 & 1.39 & - \\
\hline $\mathrm{C} 22-\mathrm{C} 23$ & 1.39 & 1.39 & 1.36 & 1.40 & 1.42 & 1.40 & - \\
\hline C23-C24 & 1.41 & 1.41 & 1.36 & 1.41 & 1.36 & 1.41 & - \\
\hline C24-C25 & 1.39 & 1.41 & 1.39 & 1.42 & 1.42 & 1.41 & - \\
\hline C25-C26 & 1.35 & 1.41 & 1.37 & 1.40 & 1.41 & 1.40 & - \\
\hline C21-C26 & 1.37 & 1.40 & 1.41 & 1.40 & 1.41 & 1.40 & - \\
\hline C31-C32 & 1.42 & 1.41 & 1.40 & 1.40 & 1.42 & 1.40 & - \\
\hline C32-C33 & 1.39 & 1.39 & 1.42 & 1.39 & 1.40 & 1.40 & - \\
\hline
\end{tabular}


Tableau 4: Suite 1.

\begin{tabular}{|c|c|c|c|c|c|c|c|}
\hline \multirow{2}{*}{ Liaisons (Å) } & \multicolumn{2}{|c|}{ Procyanidine B2-Diméthyle 1} & \multicolumn{2}{|c|}{ Procyanidine B2-Diméthyle 2} & \multicolumn{2}{|c|}{ Procyanidine B2-Diméthyle 3} & \multirow{2}{*}{ Exp } \\
\hline & $\begin{array}{l}\text { Dynamique } \\
\text { Moléculaire }\end{array}$ & $\begin{array}{c}\text { B3LYP/ } \\
6-311 G\end{array}$ & $\begin{array}{l}\text { Dynamique } \\
\text { Moléculaire }\end{array}$ & $\begin{array}{c}\text { B3LYP/ } \\
6-311 G\end{array}$ & $\begin{array}{l}\text { Dynamique } \\
\text { Moléculaire }\end{array}$ & $\begin{array}{c}\text { B3LYP/ } \\
6-311 G\end{array}$ & \\
\hline C33-C34 & 1.33 & 1.41 & 1.41 & 1.40 & 1.40 & 1.40 & - \\
\hline $\mathrm{C} 24-\mathrm{C} 25$ & 1.40 & 1.39 & 1.38 & 1.39 & 1.41 & 1.40 & - \\
\hline $\mathrm{C} 35-\mathrm{C} 36$ & 1.43 & 1.40 & 1.42 & 1.40 & 1.39 & 1.39 & - \\
\hline C31-C36 & 1.36 & 1.40 & 1.37 & 1.41 & 1.45 & 1.40 & - \\
\hline $\mathrm{C} 5-\mathrm{O} 7$ & 1.54 & 1.41 & 1.51 & 1.41 & 1.49 & 1.41 & 1.43 \\
\hline C6-C10 & 1.60 & 1.52 & 1.57 & 1.53 & 1.52 & 1.52 & 1.50 \\
\hline $\mathrm{C} 9-\mathrm{C} 10$ & 1.58 & 1.56 & 1.47 & 1.56 & 1.56 & 1.55 & 1.54 \\
\hline C8-C9 & 1.54 & 1.53 & 1.53 & 1.53 & 1.54 & 1.53 & - \\
\hline $\mathrm{C} 8-\mathrm{O} 7$ & 1.40 & 1.50 & 1.43 & 1.49 & 1.43 & 1.50 & 1.43 \\
\hline $\mathrm{C} 25-\mathrm{O} 27$ & 1.53 & 1.39 & 1.53 & 1.40 & 1.55 & 1.39 & - \\
\hline $\mathrm{C} 26-\mathrm{C} 30$ & 1.51 & 1.52 & 1.52 & 1.51 & 1.53 & 1.51 & 1.50 \\
\hline C29-C30 & 1.52 & 1.53 & 1.51 & 1.52 & 1.53 & 1.53 & 1.54 \\
\hline C28-C29 & 1.48 & 1.53 & 1.56 & 1.54 & 1.58 & 1.54 & - \\
\hline $\mathrm{C} 28-\mathrm{O} 27$ & 1.39 & 1.48 & 1.40 & 1.48 & 1.47 & 1.48 & 1.43 \\
\hline \multicolumn{8}{|c|}{ Angles de liaisons $\left(^{\circ}\right)$} \\
\hline C6-C1-C2 & 118.60 & 122.30 & 119.20 & 122.39 & 121.75 & 122.42 & 120 \\
\hline $\mathrm{C} 1-\mathrm{C} 2-\mathrm{C} 3$ & 121.77 & 118.80 & 119.65 & 118.93 & 118.46 & 118.80 & - \\
\hline $\mathrm{C} 2-\mathrm{C} 3-\mathrm{C} 4$ & 119.05 & 121.06 & 120.62 & 120.98 & 119.65 & 121.03 & - \\
\hline $\mathrm{C} 3-\mathrm{C} 4-\mathrm{C} 5$ & 119.34 & 118.57 & 119.89 & 118.49 & 119.53 & 118.56 & - \\
\hline C4-C5-C6 & 121.35 & 122.59 & 123.32 & 122.83 & 122.60 & 122.62 & - \\
\hline C5-C6-C1 & 119.60 & 116.67 & 117.02 & 116.38 & 117.24 & 116.56 & - \\
\hline
\end{tabular}


K. D. YAPO et al. /Int. J. Biol. Chem. Sci. 8(1): 355-376, 2014

Tableau 4: Suite 2.

\begin{tabular}{|c|c|c|c|c|c|c|c|}
\hline \multirow{2}{*}{ Angles de liaisons $\left(^{\circ}\right)$} & \multicolumn{2}{|c|}{ Procyanidine B2-Diméthyle 1} & \multicolumn{2}{|c|}{ Procyanidine B2-Diméthyle 2} & \multicolumn{2}{|c|}{ Procyanidine B2-Diméthyle 3} & \multirow{2}{*}{ Exp. } \\
\hline & $\begin{array}{l}\text { Dynamique } \\
\text { Moléculaire }\end{array}$ & $\begin{array}{c}\text { B3LYP/ } \\
6-311 G\end{array}$ & $\begin{array}{l}\text { Dynamique } \\
\text { Moléculaire }\end{array}$ & $\begin{array}{c}\text { B3LYP/ } \\
6-311 G\end{array}$ & $\begin{array}{l}\text { Dynamique } \\
\text { Moléculaire }\end{array}$ & $\begin{array}{c}\text { B3LYP/ } \\
6-311 G\end{array}$ & \\
\hline C16-C11-C12 & 119.90 & 118.79 & 118.93 & 118.35 & 120.38 & 119.05 & - \\
\hline C11-C12-C13 & 118.57 & 120.51 & 119.97 & 120.48 & 117.80 & 120.66 & - \\
\hline C12-C13-C14 & 121.19 & 120.08 & 119.43 & 120.74 & 121.66 & 120.02 & - \\
\hline C13-C14-C15 & 120.95 & 120.05 & 123.02 & 119.35 & 118.26 & 119.90 & - \\
\hline C14-C15-1C6 & 116.22 & 119.89 & 118.05 & 119.33 & 120.95 & 120.33 & - \\
\hline C15-C16-C11 & 122.61 & 120.64 & 119.92 & 121.75 & 120.26 & 120.02 & - \\
\hline $\mathrm{C} 26-\mathrm{C} 21-\mathrm{C} 22$ & 120.41 & 121.63 & 119.20 & 121.66 & 120.24 & 121.61 & - \\
\hline $\mathrm{C} 21-\mathrm{C} 22-\mathrm{C} 23$ & 121.74 & 118.87 & 119.45 & 118.70 & 118.45 & 118.70 & - \\
\hline C22-C23-C24 & 118.51 & 122.89 & 120.23 & 122.80 & 122.73 & 122.83 & - \\
\hline $\mathrm{C} 23-\mathrm{C} 24-\mathrm{C} 25$ & 117.36 & 115.94 & 120.54 & 115.95 & 118.38 & 115.96 & - \\
\hline $\mathrm{C} 24-\mathrm{C} 25-\mathrm{C} 26$ & 122.52 & 123.06 & 121.61 & 122.87 & 120.09 & 122.93 & - \\
\hline C25-C26-C21 & 118.83 & 117.59 & 117.67 & 117.85 & 119.82 & 117.88 & - \\
\hline C36-C31-C32 & 121.65 & 119.23 & 124.84 & 118.55 & 117.82 & 118.78 & - \\
\hline C31-C32-C33 & 121.96 & 120.50 & 117.74 & 120.26 & 120.14 & 120.85 & - \\
\hline C32-C33-C34 & 116.03 & 119.52 & 118.80 & 121.00 & 118.48 & 120.24 & - \\
\hline C33-C34-C35 & 123.33 & 120.14 & 120.07 & 119.26 & 124.86 & 118.91 & - \\
\hline C34-C35-C36 & 121.24 & 120.15 & 122.17 & 120.11 & 115.52 & 120.99 & - \\
\hline C35-C36-C31 & 115.19 & 120.36 & 115.99 & 120.79 & 122.78 & 120.21 & - \\
\hline \multicolumn{8}{|l|}{ Angles dièdre $\varphi\left(^{\circ}\right)$} \\
\hline C9-C10-C24-C25 & 92.33 & 92.50 & 91.58 & 88.31 & 88.62 & 93.37 & \pm 90 \\
\hline
\end{tabular}


K. D. YAPO et al. / Int. J. Biol. Chem. Sci. 8(1): 355-376, 2014

Tableau 4: Suite 3.

\begin{tabular}{|c|c|c|c|c|c|c|c|}
\hline \multirow{2}{*}{ Liaisons (Å) } & \multicolumn{2}{|c|}{ Procyanidine B2-Diméthyle 4} & \multicolumn{2}{|c|}{ Procyanidine B2-Diméthyle 5} & \multicolumn{2}{|c|}{ Procyanidine B2-Diméthyle 6} & \multirow[b]{2}{*}{ Exp. } \\
\hline & $\begin{array}{l}\text { Dynamique } \\
\text { Moléculaire }\end{array}$ & $\begin{array}{l}\text { B3LYP/ } \\
\text { 6-311G }\end{array}$ & $\begin{array}{l}\text { Dynamique } \\
\text { Moléculaire }\end{array}$ & $\begin{array}{l}\text { B3LYP/ } \\
\text { 6-311G }\end{array}$ & $\begin{array}{l}\text { Dynamique } \\
\text { Moléculaire }\end{array}$ & $\begin{array}{l}\text { B3LYP/ } \\
\text { 6-311G }\end{array}$ & \\
\hline $\mathrm{C} 1-\mathrm{C} 2$ & 1.45 & 1.39 & 1.39 & 1.39 & 1.38 & 1.39 & 1.40 \\
\hline $\mathrm{C} 2-\mathrm{C} 3$ & 1.38 & 1.40 & 1.44 & 1.39 & 1.41 & 1.39 & - \\
\hline C3-C4 & 1.41 & 1.40 & 1.38 & 1.40 & 1.41 & 1.39 & - \\
\hline $\mathrm{C} 4-\mathrm{C} 5$ & 1.38 & 1.40 & 1.36 & 1.40 & 1.38 & 1.40 & - \\
\hline C5-C6 & 1.36 & 1.40 & 1.44 & 1.40 & 1.40 & 1.40 & - \\
\hline C1-C6 & 1.41 & 1.41 & 1.44 & 1.41 & 1.46 & 1.41 & - \\
\hline C11-C12 & 1.43 & 1.40 & 1.40 & 1.40 & 1.44 & 1.40 & - \\
\hline $\mathrm{C} 12-\mathrm{C} 13$ & 1.41 & 1.39 & 1.40 & 1.40 & 1.37 & 1.40 & - \\
\hline C13-C14 & 1.38 & 1.40 & 1.40 & 1.39 & 1.37 & 1.40 & - \\
\hline C14-C15 & 1.38 & 1.41 & 1.41 & 1.41 & 1.34 & 1.41 & - \\
\hline C15-C16 & 1.36 & 1.40 & 1.42 & 1.39 & 1.40 & 1.39 & - \\
\hline C11-C16 & 1.44 & 1.40 & 1.40 & 1.41 & 1.39 & 1.40 & - \\
\hline C21-C22 & 1.39 & 1.39 & 1.39 & 1.39 & 1.42 & 1.39 & - \\
\hline C22-C23 & 1.37 & 1.40 & 1.40 & 1.40 & 1.40 & 1.39 & - \\
\hline C23-C24 & 1.40 & 1.41 & 1.38 & 1.41 & 1.42 & 1.40 & - \\
\hline C24-C25 & 1.35 & 1.41 & 1.36 & 1.41 & 1.38 & 1.41 & - \\
\hline C25-C26 & 1.41 & 1.40 & 1.37 & 1.405 & 1.41 & 1.40 & - \\
\hline C21-C26 & 1.36 & 1.40 & 1.42 & 1.40 & 1.39 & 1.40 & - \\
\hline C31-C32 & 1.45 & 1.40 & 1.41 & 1.40 & 1.38 & 1.40 & - \\
\hline C32-C33 & 1.42 & 1.39 & 1.41 & 1.39 & 1.44 & 1.39 & - \\
\hline C33-C34 & 1.39 & 1.40 & 1.40 & 1.40 & 1.39 & 1.40 & - \\
\hline
\end{tabular}


K. D. YAPO et al. / Int. J. Biol. Chem. Sci. 8(1): 355-376, 2014

Tableau 4: Suite 4.

\begin{tabular}{|c|c|c|c|c|c|c|c|}
\hline \multirow{2}{*}{ Liaisons (§) } & \multicolumn{2}{|c|}{ Procyanidine B2-Diméthyle 4} & \multicolumn{2}{|c|}{ Procyanidine B2-Diméthyle 5} & \multicolumn{2}{|c|}{ Procyanidine B2-Diméthyle 6} & \multirow[b]{2}{*}{ Exp. } \\
\hline & $\begin{array}{l}\text { Dynamique } \\
\text { Moléculaire }\end{array}$ & $\begin{array}{l}\text { B3LYP/ } \\
\text { 6-311G }\end{array}$ & $\begin{array}{l}\text { Dynamique } \\
\text { Moléculaire }\end{array}$ & $\begin{array}{l}\text { B3LYP/ } \\
\text { 6-311G }\end{array}$ & $\begin{array}{l}\text { Dynamique } \\
\text { Moléculaire }\end{array}$ & $\begin{array}{l}\text { B3LYP/ } \\
\text { 6-311G }\end{array}$ & \\
\hline $\mathrm{C} 24-\mathrm{C} 25$ & 1.37 & 1.40 & 1.39 & 1.40 & 1.32 & 1.40 & - \\
\hline C35-C36 & 1.38 & 1.39 & 1.42 & 1.39 & 1.41 & 1.39 & - \\
\hline C31-C36 & 1.42 & 1.40 & 1.43 & 1.40 & 1.46 & 1.40 & - \\
\hline $\mathrm{C5-O7}$ & 1.55 & 1.41 & 1.51 & 1.41 & 1.54 & 1.40 & 1.43 \\
\hline C6-C10 & 1.55 & 1.52 & 1.55 & 1.50 & 1.56 & 1.52 & 1.50 \\
\hline C9-C10 & 1.55 & 1.56 & 1.55 & 1.55 & 1.52 & 1.55 & 1.54 \\
\hline $\mathrm{C} 8-\mathrm{C} 9$ & 1.50 & 1.53 & 1.50 & 1.53 & 1.52 & 1.54 & - \\
\hline $\mathrm{C} 8-\mathrm{O} 7$ & 1.45 & 1.50 & 1.47 & 1.50 & 1.41 & 1.46 & 1.43 \\
\hline $\mathrm{C} 25-\mathrm{O} 27$ & 1.55 & 1.39 & 1.56 & 1.39 & 1.54 & 1.40 & - \\
\hline C26-C30 & 1.57 & 1.51 & 1.44 & 1.51 & 1.55 & 1.51 & 1.50 \\
\hline C29-C30 & 1.53 & 1.53 & 1.51 & 1.53 & 1.54 & 1.52 & 1.54 \\
\hline C28-C29 & 1.48 & 1.54 & 1.54 & 1.54 & 1.52 & 1.54 & - \\
\hline C28-O27 & 1.40 & 1.48 & 1.44 & 1.48 & 1.42 & 1.48 & 1.43 \\
\hline \multicolumn{8}{|c|}{ Angles de liaisons $\left(^{\circ}\right)$} \\
\hline C6-C1-C2 & 121.06 & 122.43 & 118.55 & 122.45 & 120.46 & 122.08 & 120 \\
\hline C1-C2-C3 & 118.62 & 118.76 & 121.93 & 118.72 & 119.75 & 118.98 & - \\
\hline C2-C3-C4 & 120.86 & 121.05 & 118.77 & 121.05 & 120.41 & 121.02 & - \\
\hline C3-C4-C5 & 117.85 & 118.59 & 119.97 & 118.65 & 119.54 & 118.80 & - \\
\hline C4-C5-C6 & 125.50 & 122.55 & 123.38 & 122.44 & 122.07 & 122.31 & - \\
\hline C5-C6-C1 & 116.07 & 116.60 & 117.13 & 116.67 & 117.69 & 116.64 & - \\
\hline
\end{tabular}


K. D. YAPO et al. / Int. J. Biol. Chem. Sci. 8(1): 355-376, 2014

Tableau 4: Suite 5.

\begin{tabular}{|c|c|c|c|c|c|c|c|}
\hline \multirow[t]{2}{*}{ Angles de liaisons $\left({ }^{\circ}\right)$} & \multicolumn{2}{|c|}{ Procyanidine B2-Diméthyle 4} & \multicolumn{2}{|c|}{ Procyanidine B2-Diméthyle 5} & \multicolumn{2}{|c|}{ Procyanidine B2-Diméthyle 6} & \multirow[b]{2}{*}{ Exp. } \\
\hline & $\begin{array}{l}\text { Dynamique } \\
\text { Moléculaire }\end{array}$ & $\begin{array}{c}\text { B3LYP/ } \\
\text { 6-311G }\end{array}$ & $\begin{array}{l}\text { Dynamique } \\
\text { Moléculaire }\end{array}$ & $\begin{array}{c}\text { B3LYP/ } \\
6-311 G\end{array}$ & $\begin{array}{l}\text { Dynamique } \\
\text { Moléculaire }\end{array}$ & $\begin{array}{c}\text { B3LYP/ } \\
6-311 G\end{array}$ & \\
\hline C16-C11-C12 & 119.93 & 118.46 & 120.94 & 118.97 & 119.53 & 118.95 & - \\
\hline C11-C12-C13 & 117.71 & 119.74 & 118.34 & 120.69 & 118.24 & 119.32 & - \\
\hline C12-C13-C14 & 119.41 & 121.71 & 122.401 & 119.99 & 120.71 & 121.66 & - \\
\hline $\mathrm{C} 13-\mathrm{C} 14-\mathrm{C} 15$ & 124.17 & 118.90 & 119.00 & 119.82 & 122.04 & 119.03 & - \\
\hline C14-C15-1C6 & 118.00 & 119.15 & 118.53 & 120.45 & 120.07 & 119.06 & - \\
\hline C15-C16-C11 & 120.70 & 122.03 & 119.07 & 120.09 & 119.39 & 121.96 & - \\
\hline $\mathrm{C} 26-\mathrm{C} 21-\mathrm{C} 22$ & 119.41 & 121.48 & 116.02 & 121.58 & 117.52 & 121.25 & - \\
\hline C21-C22-C23 & 120.30 & 118.78 & 121.84 & 118.72 & 122.42 & 118.41 & - \\
\hline C22-C23-C24 & 120.89 & 122.86 & 119.87 & 122.79 & 119.34 & 123.85 & - \\
\hline $\mathrm{C} 23-\mathrm{C} 24-\mathrm{C} 25$ & 118.04 & 115.80 & 118.32 & 115.95 & 117.93 & 115.04 & - \\
\hline C24-C25-C26 & 121.99 & 123.08 & 121.61 & 122.95 & 122.75 & 123.27 & - \\
\hline $\mathrm{C} 25-\mathrm{C} 26-\mathrm{C} 21$ & 119.30 & 117.92 & 120.53 & 117.83 & 119.88 & 118.10 & - \\
\hline C36-C31-C32 & 120.38 & 118.75 & 120.21 & 118.70 & 118.94 & 118.44 & - \\
\hline C31-C32-C33 & 116.75 & 120.40 & 115.64 & 121.08 & 116.07 & 120.87 & - \\
\hline C32-C33-C34 & 117.63 & 120.92 & 126.70 & 119.70 & 123.90 & 120.28 & - \\
\hline C33-C34-C35 & 127.06 & 118.53 & 115.91 & 119.61 & 119.64 & 119.22 & - \\
\hline C34-C35-C36 & 116.09 & 120.86 & 121.52 & 120.35 & 119.52 & 120.36 & - \\
\hline C35-C36-C31 & 121.34 & 120.51 & 119.52 & 120.54 & 121.35 & 120.77 & - \\
\hline \multicolumn{8}{|l|}{ Angles dièdre $\varphi\left(^{\circ}\right)$} \\
\hline C9-C10-C24-C25 & 87.05 & 92.71 & $-83,87$ & 95.80 & 89.96 & 61.831 & \pm 90 \\
\hline
\end{tabular}


Les longueurs expérimentales de liaisons C-C et angles de liaisons C-C-C des groupements phényles sont respectivement de l'ordre $1.40 \AA$ et $120^{\circ}$ (Fox et al., 1995). Les longueurs de liaisons des modèles issus de la Dynamique Moléculaire présentent des écarts plus ou moins importants quand à l'observation. Ainsi, on constate pour le Procyanidine B2-Diméthyle 1, des écarts allant jusqu'à $0.07 \AA$ en dessous de $1.40 \AA$ et $0.04 \AA$ au dessus de $1.40 \AA$. Ces écarts sont respectivement de $0.05 \AA$ et de $0.04 \AA$ pour le Procyanidine B2-Diméthyle 2, de $0.06 \AA$ et de $0.05 \AA$ pour le Procyanidine B2-Diméthyle 3, de $0.05 \AA$ et de $0.05 \AA$ pour le Procyanidine B2-Diméthyle 4, de $0.04 \AA$ et de $0.04 \AA$ pour le Procyanidine B2-Diméthyle 5 et enfin de $0.08 \AA$ et de $0.06 \AA$ pour le Procyanidine B2Diméthyle 6. Quand aux modèles de Mécanique Quantique (B3LYP/6-311G), les écarts sont de \pm de $0.01 \AA$ pour tous. Ainsi on a, au niveau des modèles de Dynamique Moléculaire, la longueur de liaison C-O accolée au cycle benzénique ont des variations allant jusqu'à $0.12 \AA$ au dessus de l'observation (1.43 $\AA$ ) alors que l'autre liaison $\mathrm{C}-\mathrm{O}$ varie relativement moins $( \pm 0.04 \AA$ par rapport à l'observation). Quand aux modèles de la Mécanique Quantique, la longueur de liaison $\mathrm{C}-\mathrm{O}$ accolée au cycle benzénique varie plutôt de $-0.04 \AA$ par rapport à l'observation pendant que l'autre liaison $\mathrm{C}$-O varie jusqu'à $0.07 \AA$ au dessus de l'observation. Enfin, concernant les liaisons simples C-C des cycles pyrannes des différents modèles, on observe des variations allant de -0.07 à $+0.10 \AA$ au maximum quand aux données expérimentales. Cette observation est faite pour les modèles de Mécanique Quantique et de Dynamique Moléculaire mais un peu plus prononcée (variation) pour cette dernière méthode de calcul.

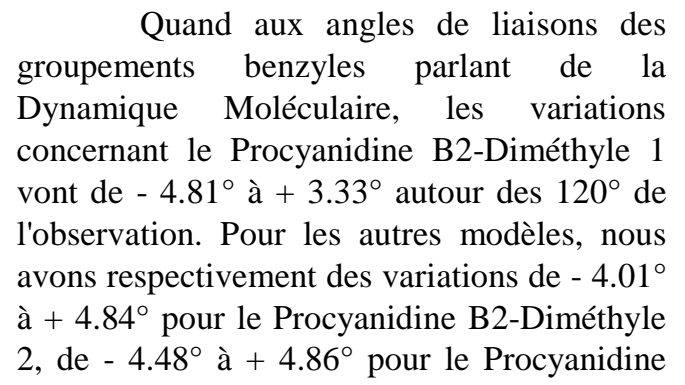

B2-Diméthyle 3 , de $-3.93^{\circ}$ à $+7.06^{\circ}$ pour le Procyanidine B2-Diméthyle 4 , de $-4.36^{\circ}$ à + $6.70^{\circ}$ pour le Procyanidine B2-Diméthyle 5 et de $-3.93^{\circ}$ à $+3.90^{\circ}$ pour le Procyanidine B2Diméthyle 6. Pour les modèles de Mécanique Quantique (B3LYP/6-311G), nous avons des variations de $-4.06^{\circ}$ à $+3.60^{\circ}$ pour le Procyanidine B2-Diméthyle 1, - 4.05 à + $2.83^{\circ}$ pour le Procyanidine B2-Diméthyle 2, $4.04^{\circ}$ à $+2.93^{\circ}$ pour le Procyanidine B2Diméthyle $3,-4.20^{\circ}$ à $+3.08^{\circ}$ pour le Procyanidine B2-Diméthyle $4,-4.05^{\circ}$ à + $2.95^{\circ}$ pour le Procyanidine B2-Diméthyle 5 et - $4.96^{\circ}$ à $+3.85^{\circ}$ pour le Procyanidine B2Diméthyle 6. Ces résultats nous indiquent clairement que les variations autour de la valeur expérimentale $\left(120^{\circ}\right)$ sont plus importantes pour les modèles de Dynamique Moléculaire par rapport à ceux de Mécanique Quantique.

La variation des angles dièdres des modèles de Dynamique Moléculaire couplée à ceux de la Mécanique Quantique (B3LYP/6311G) est présentée au niveau de la Figure 4. On constate que les modèles Procyanidine B2Diméthyle 1, Procyanidine B2-Diméthyle 2, Procyanidine B2-Diméthyle 3 et Procyanidine B2-Diméthyle 4 de Dynamique Moléculaire et de Mécanique Quantique ont des angles dièdres autour de $90^{\circ}$. Ce qui voudrait dire que ces modèles ont des angles pendant la Dynamique Moléculaire comme la Mécanique Quantique qui varient dans le même ordre. Ce qui nous donne pour le Procyanidine B2Diméthyle $192,33^{\circ}$ en Dynamique Moléculaire et $92.50^{\circ}$ en Mécanique Quantique. Nous avons ensuite respectivement $91,58^{\circ}$ et $88.31^{\circ}$ pour le Procyanidine B2-Diméthyle 2, 88,62 ${ }^{\circ}$ et $93.37^{\circ}$ pour le Procyanidine B2-Diméthyle 3 et enfin $87,05^{\circ}$ et $92.71^{\circ}$ pour le Procyanidine B2-Diméthyle 4. Le Procyanidine B2Diméthyle 5 et le Procyanidine B2-Diméthyle 6 ne rentrent pas dans ce schéma en ne présentant pas de valeurs similaires. Nous avons respectivement en Dynamique Moléculaire et en Mécanique Quantique $83,87^{\circ}$ et $95.80^{\circ}$ pour le Procyanidine B2Diméthyle 5 et $89,96^{\circ}$ et $61.83^{\circ}$ pour le Procyanidine B2-Diméthyle 6. 


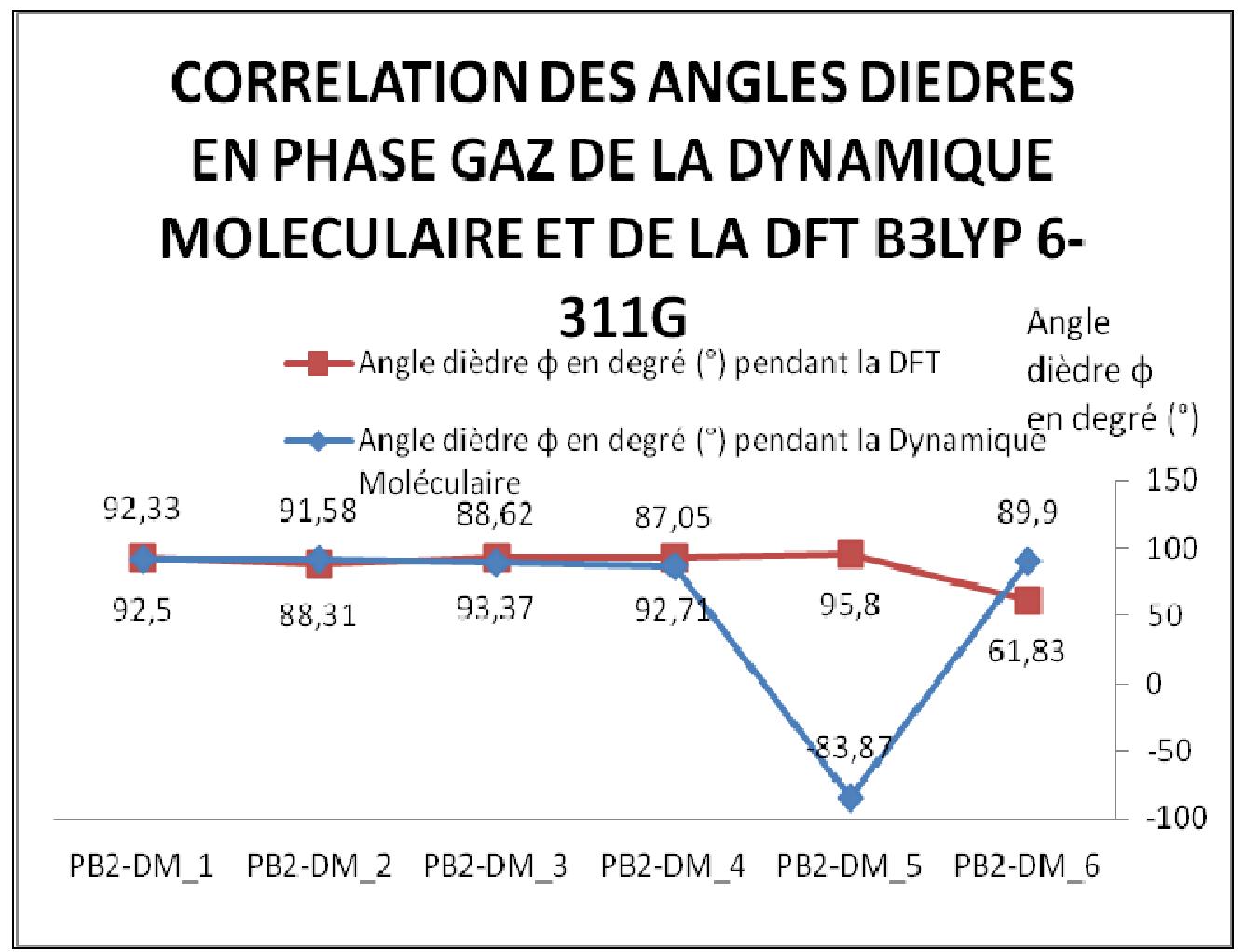

Figure 13: Corrélation en phase gaz des angles dièdres en Dynamique Moléculaire et en DFT B3LYP/6-311G.

\section{DISCUSSION}

Les résultats des simulations à l'aide du champ de forces SPASIBA montrent que les modèles Procyanidine B2-Diméthylés au niveau des groupements hydroxyles consécutifs d'un seul monomère (Procyanidine B2-Diméthyle 1 et Procyanidine B2-Diméthyle 2) sont dans de meilleures dispositions que les modèles avec une méthylation sur un monomère et l'autre méthylation sur le second monomère (Procyanidine B2-Diméthyle 3, Procyanidine B2-Diméthyle 4, Procyanidine B2-Diméthyle 5) à l'exception du Procyanidine B2Diméthyle 6.

Se référant aux données des longueurs de liaisons, des angles de liaisons et des angles dièdres (en dynamique moléculaire et en mécanique quantique), on pourrait dire que les déformations des cycles benzéniques au niveau des différents modèles sont plus prononcées en Dynamique Moléculaire par rapport à la mécanique quantique. Ces déformations des cycles benzéniques induisent des déformations aussi marquées sur les cycles pyrannes des différents modèles surtout en Dynamique Moléculaire.

Par rapport à l'effet Jahn Teller (Toute molécule non linéaire dans un état électronique dégénéré (orbitale ou spin) se déforme de manière à diminuer sa symétrie, lever la dégénérescence et minimiser son énergie), nous pouvons dire que les modèles de la Dynamique Moléculaire seraient plus 
représentatifs de la stabilité que ceux de la Mécanique Quantique car présentant plus de déformations. Ce qui nous amène à privilégier les résultats de la Dynamique Moléculaire. Par rapport à cela, nous pourrions affirmer qu'au vue des Tableaux 1 à 4, des Figures 9, 12 et 13, que les modèles 1 et 2 sont ceux qui gardent une géométrie proche de celle l'expérimentation quant aux valeurs de leur angle dièdre quelle que soit la méthode de calcul en phase gaz (Champ de Forces SPASIBA et B3LYP/6-311G).

\section{CONCLUSION}

$\mathrm{Au}$ terme de cette étude, nous pourrons dire que les molécules de Procyanidine B2 $O$-diméthylées que Seigo et son équipe ont mis en évidence dans le milieu intérieur (plasma) seraient principalement les modèles Procyanidine B2-Diméthylés au niveau des groupements hydroxyles consécutifs d'un seul monomère (Procyanidine B2-Diméthyle 1 et Procyanidine B2-Diméthyle 2) qui seraient dans de meilleures dispositions que les modèles avec une méthylation sur un monomère et l'autre méthylation sur le second monomère. On pourrait donc proposer ces modèles à synthétiser et par marquage radioactif, suivre leur évolution depuis la bouche jusqu'au milieu intérieur (plasma). Ceci nous permettrait de vérifier leur affinité par rapport au fer non héminique $\left(\mathrm{Fe}^{3+}, \mathrm{Fe}^{2+}\right)$ durant leur trajet digestif.

\section{REFERENCES}

Bors W, Michel C, Stettmaier K. 2000. Electron paramagnetic resonance studies of radical species of proanthocyanidins and gallate esters. Archives of Biochemistry and Biophysics, 374(2): 347-355.

Brune M, Hallberg L, Skanberg AB. 1991. Determination of iron-binding phenolic groups in foods. Journal of Food Science, 56(1): 128-131.

De Freitas VAP, Glories Y, Monique A. 2000. Developmental changes of procyanidins in grapes of red Vitis vinifera varieties and their composition in respective wines. American Journal of Enology and Viticulture, 5(14): 397-403.

Disler PB, Lynch SR, Charlton RW, Torrance JD, Bothwell TH, Walker RB, Mayet F. 1975. The effect of tea on iron absorption. Gut, 16: 193-200.

Fox, Anne M, Whitesell, James K. 1995. Organische Chemie: Grundlagen, Mechanismen, Bioorganische Anwendungen. Springer.

Jeremy PES. 2003. Metabolism of tea flavonoids in the gastrointestinal tract. American Society for Nutritional Sciences, 133(10): 3255S-3261S.

Khan ML, Haslam E, Williamson MP. 1997. Structure and conformation of the Procyanidin B-2 Dimer. Magnetic Resonance in Chemistry, 35(12): 854858.

Manach C, Scalbert A, Morand C, Remesy C, Jimenez L. 2004. Polyphenols: food sources and bioavailability. American Journal of Clinical Nutrition, 79: 727747.

Roberta RH, Sheryl AL, Cameron MS, Qin YZ, Derek DS, John FH, Cesar GF, Harold HS, Carl LK. 2002. Procyanidin dimer B2 [epicatechin-( $4 \beta-8)$ epicatechin] in human plasma after the consumption of a flavanol-rich cocoa. American Journal of Clinical Nutrition, 76(4): 798-804.

Scalbert A, Morand C, Manach C, Rémésy C. 2002. Absorption and metabolism of polyphenols in the gut and impact on health. Biomedicine Pharmarcotherapy, 56(6): 276-282.

Seigo B, Naomi O, Midori N, Junji T. 2002. Absorption and urinary excretion of 
procyanidin B2 [epicatechin- $(4 \beta-8)$ epicatechin] in rats. Free Radical Biology and Medicine, 33(1): 142-148.

Still WC, Tempczyk A, Hawley RC, Hendrickson TF. 1990. Semi analytical treatment of solvation for molecular mechanics and dynamics. Journal of the American Chemical Society, 112(16): 6127-6129.

Tapiero H, Tew KD, Ba GN, Mathe G. 2002. Polyphenols: do they play a role in the prevention of human pathologies? Biomedicine Pharmacotherapy, 56(4): 200-207.
Yapo KD, Lagant P, Vergoten G. 2008. Empirical Modelling of Nonheminic Iron-Tannins Complexes By Spasiba Forces Field: Case of $\mathrm{Fe}^{3+/ 2+}$-Procyanidin B2 Complexes. Revue Ivoirienne des Sciences et Technologies, 11: 27-43.

Yapo KD, Lagant P, Vergoten G. 2007. The SPASIBA Force Field for Studying IronTannins Interactions: Application to $\mathrm{Fe}^{3+} / \mathrm{Fe}^{2+} \quad$ Catechol Complexes. International Journal of Molecular Sciences, 8: 259-272. 